DEMONSTRATIO MATHEMATICA

Vol. XXVIII No $2 \quad 1995$

Jan S. Rogulski

\title{
ON SYMMETRIC MOTIONS OF A BAROTROPIC FLUID
}

\section{Introduction}

The initial value problem which corresponds to a motion of an inviscid barotropic fluid in all of $R^{n}$ has a unique solution in a suitable space of curves in Sobolev spaces (for existence and uniqueness theorems proven by Kato see [2]). In $1979 \mathrm{D}$. Ebin proved that the equations of motion in a bounded domain have a solution on some time interval provided that the initial fluid density is nearly constant and the initial fluid velocity is everywhere less than the sound speed. The solutions of initial problems are constructed as limits of solutions $U_{n}$ of some other (in general, linearized) systems. In the next step of recursion, $U_{n+1}$ is a solution of a system, whose coefficients depend on $U_{n}$. The main part of proofs is devoted to obtain an estimate $\left\|U_{n+1}-U_{n}\right\| \leq \alpha\left\|U_{n}-U_{n-1}\right\|$, for some $\alpha<1$, in an appropriate norm. This is very difficult and requires some rearrangements of the initial problem at the very beginning, in particular, the equations of motion are first transformed and written as a quasilinear symmetric hyperbolic system, or considered in a quite different, but equivalent, form (see Ebin [1]).

It seems to be impossible to study the local behaviour of solutions by means of representing them in the form of such limits. In this paper we describe a simple version of a method, by means of which one can represent solutions of some quasilinear systems in implicit form. In Section 2 there are two examples illustrating the efficiency of this method for some specific systems. Then we apply this method to equations of symmetric motions in a ball in $R^{n}$. It turns out that the non-linear nature of barotropic motions is so deep, that one can obtain only a very small set of solutions by means of that method. But we still hope that further refinements of the method would lead to some convenient representation of solutions. We also show that all symmetric motions are generated by solutions of a single second order quasilinear hyperbolic equation with an unknown real function of two variables. 


\section{Solutions defined implicitly}

We first discuss a method of finding classical solutions of some kind of nonlinear systems of partial differential equations. Let $G: R^{m} \rightarrow R^{n}$ be a given function of class $C^{2}$. We are looking for functions $U: R^{2} \supset \Omega \ni$ $(t, y) \mapsto U(t, y) \in R^{m}$ of class $C^{1}$ satisfying the following equation

$$
\frac{\partial U}{\partial t}=\frac{\partial}{\partial y}(G \circ U)
$$

that is, we deal with systems of the form

$$
\left\{\begin{aligned}
\frac{\partial u_{1}}{\partial t} & =\frac{\partial}{\partial y} g_{1}\left(u_{1}(t, y), \ldots, u_{m}(t, y)\right) \\
\vdots & \\
\frac{\partial u_{m}}{\partial t} & =\frac{\partial}{\partial y} g_{m}\left(u_{1}(t, y), \ldots, u_{m}(t, y)\right),
\end{aligned}\right.
$$

where $G(u)=\left(g_{1}(u), \ldots, g_{m}(u)\right)$ for $u \in R^{m}$ and $U(t, y)=\left(u_{1}(t, y), \ldots\right.$, $\left.\ldots, u_{m}(t, y)\right),(t, y) \in \Omega \subset R^{2}$.

We assume that the system (1.1) is in a sense "hyperbolic", namely, that at any point $u \in R^{m}$ the eigenvalues of the Jacobian $D G(u)$ are real and, moreover, for every $u \in R^{m}$ there exists a basis of $R^{m}$ consisting of eigenvectors of $D G(u)$. Since $G$ is of class $C^{2}$, we may further assume that these eigenvalues and eigenvectors are organized as suitable functions of class $C^{1}$, i.e., there exist functions $\lambda_{1}, \ldots, \lambda_{m} \in C^{1}\left(R^{m}, R\right)$ and vectorfields $V_{1}, \ldots, V_{m} \in C^{1}\left(R^{m}, R^{m}\right)$ such that

$$
D G(u) \cdot V_{j}(u)=\lambda_{j}(u) V_{j}(u)
$$

for any $j=1, \ldots, m$ and for every $u \in R^{m}$.

Every solution of class $C^{1}$ of the system (1.1) defined on $\Omega$ may be viewed in the implicit form $H(t, y, u)=C$ for some function $H \in C^{1}\left(\Omega \times R^{m}, R^{m}\right)$ and $C \in R^{m}$, where $H(t, y, \cdot): R^{m} \rightarrow R^{m}$ is a $C^{1}$-diffeomorphism, in particular, $D_{u} H(t, y, u): R^{m} \rightarrow R^{m}$ is invertible.

This can be done in several ways and, in fact, even if we put some additional conditions on $D_{u} H$, the freedom of choice of a function $H$ defining the same functions implicitly is still very large.

By virtue of the Implicit Function Theorem, we have

$$
\frac{\partial U}{\partial t}=-\left(D_{u} H\right)^{-1} \cdot \frac{\partial H}{\partial t} \text { and } \frac{\partial U}{\partial y}=-\left(D_{u} H\right)^{-1} \cdot \frac{\partial H}{\partial y}
$$

for a function $U$ defined by $H$. Substituting this into the system (1.1), we obtain a condition for $H$ generating solutions of this system

$$
\frac{\partial H}{\partial t}=\left(D_{u} H\right) \cdot D G \cdot\left(D_{u} H\right)^{-1} \frac{\partial H}{\partial y} \text {. }
$$


If we add the requirement that $D_{u} H$ and $D G$ commute, we simplify the above system to the following, linear form

$$
\begin{gathered}
\frac{\partial H}{\partial t}(t, y, u)=D G(u) \frac{\partial H}{\partial y}(t, y, u), \\
{\left[D G(u), D_{u} H(t, y, u)\right]=0 .}
\end{gathered}
$$

This is a system of $m(m+1)$ linear partial differential equations for $m$ unknown functions of $m+2$ variables. Some of the equations in (1.4.2) may be linearly dependent. Any function $H \in C^{1}\left(\Omega \times R^{m}, R^{m}\right)$ uniquely defines functions $\chi_{1}, \ldots, \chi_{m} \in C^{1}\left(\Omega \times R^{m}, R\right)$ such that

$$
H(t, y, u)=\sum_{j=1}^{m} \chi_{j}(t, y, u) V_{j}(u) .
$$

Substituting this into (1.4.1), we obtain a system of $m$ equations for $\chi_{j}$, each one being an equation with a single unknown function

$$
\frac{\partial \chi_{j}}{\partial t}(t, y, u)=\lambda_{j}(u) \frac{\partial \chi_{j}}{\partial y}(t, y, u) \quad j=1,2, \ldots, m .
$$

We can easily find characteristics for each of these equations and integrate all of them. All solutions of this system have the form

$$
\chi_{j}(t, y, u)=h_{j}\left(t \cdot \lambda_{j}(u)+y, u\right), \quad j=1, \ldots, m,
$$

where $h_{j} \in C^{1}\left(U_{j} \times R^{m}, R\right)$ is arbitrary and $U_{j} \subset R$ is an interval dependent on $\Omega$ and values of $\lambda_{j}$. Hence, the set of all solutions of the system (1.4.1) is found

$$
H(t, y, u)=\sum_{j=1}^{m} h_{j}\left(t \cdot \lambda_{j}(u)+y, u\right) V_{j}(u) .
$$

But not every function $u=U(t, y)$ defined implicitly by $I I$ described in (1.5.1) satisfies the system (1.1). The function $I$ has also to fulfil the conditions (1.4.2). The commutator $\left[D G, D_{u} H\right]$ vanishes if and only if we have $D G \cdot D_{u} H \cdot V_{i}-D_{u} H \cdot D G \cdot V_{i}=0$ for every $i$. Therefore, the system (1.4.2) is equivalent to the following system of equations for $\chi_{j}$ defined in (1.5)

$$
\sum_{j=1}^{m}\left[\left(\lambda_{j}-\lambda_{i}\right)\left(D_{u} \chi_{j} \cdot V_{i}\right) V_{j}+\chi_{j}\left(D G-\lambda_{i} I\right) D V_{j} \cdot V_{i}\right]=0
$$

$$
i=1, \ldots, m \text {. }
$$

Since $V_{1}(u), \ldots, V_{m}(u)$ is a basis of $R^{m}$, we can express vector fields $D V_{k} \cdot V_{i}$ as linear combinations of $V_{1}, \ldots, V_{m}$

$$
D V_{k}(u) \cdot V_{i}(u)=\sum_{l=1}^{m} v_{k i l}(u) V_{l}(u),
$$

where $v_{k i l}$ are uniquely defined functions of class $C^{1}$ by (known) vector fields $V_{1}, \ldots, V_{m}$. We now see that the conditions (1.5.2) are equivalent to 
the following system

$$
\left(\lambda_{j}-\lambda_{i}\right)\left(D_{u} \chi_{j} \cdot V_{i}+\sum_{k=1}^{m} v_{k i j} \chi_{k}\right)=0 \quad \text { for } i, j=1, \ldots, m .
$$

This system consists of at most $m(m-1)$ equations, if all eigenvalues have their multiplicity equal to one. Can we really put so restrictive conditions upon the function $H$ ? Notice, that for any given $C^{\mathbf{1}}$-function $U: \Omega \rightarrow R^{m}$ and for any $C^{1}$-function $W: \Omega \times R^{m} \rightarrow R^{m}$ such that $W(t, y, \cdot): R^{m} \rightarrow R^{m}$ is a $C^{1}$-diffeomorphism for every $(t, y) \in \Omega$, the function $H(t, y, u):=$ $W(t, y, u)-W(t, y, U(t, y))$ has the following properties:

(i) $H: \Omega \times R^{m} \rightarrow R^{m}$ is a $C^{1}$-function.

(ii) the map $R^{m} \ni u \rightarrow H(t, y, u) \in R^{m}$ is a $C^{1}$ diffeomorphism for every $(t, y) \in \Omega$.

(iii) $H(t, y, u)=0$ defines uniquely the function $u=U(t, y)$ on $\Omega$.

(iv) $D_{u} H=D_{u} W$.

Thus $D_{u} H$ can be prescribed arbitrarily.

If $m=2$, we obtain from (1.5.2) two equations for $\chi_{1}$ and $\chi_{2}$ :

$$
\left\{\begin{aligned}
\left(\lambda_{1}-\lambda_{2}\right)\left|V_{1}\right|^{2} D_{u} \chi_{1} \cdot V_{2}+ & \left\langle V_{1},\left(D G-\lambda_{2} I\right) D V_{1} \cdot V_{2}\right\rangle \chi_{1}+ \\
& +\left\langle V_{1},\left(D G-\lambda_{2} I\right) D V_{2} \cdot V_{2}\right\rangle \chi_{2}=0 \\
\left(\lambda_{2}-\lambda_{1}\right)\left|V_{2}\right|^{2} D_{u} \chi_{2} \cdot V_{1}+ & \left\langle V_{2},\left(D G-\lambda_{1} I\right) D V_{1} \cdot V_{1}\right\rangle \chi_{1}+ \\
& +\left\langle V_{2},\left(D G-\lambda_{1} I\right) D V_{2} \cdot V_{1}\right\rangle \chi_{2}=0
\end{aligned}\right.
$$

The brackets $\langle$,$\rangle denote the standard scalar product in R^{2}$ and $|u|^{2}=\langle u, u\rangle$, $u \in R^{2}$.

\section{Examples}

We give two simple examples to illustrate how the method described in the previous section works.

\subsection{A linear system}

Let us find all classical solutions of the system

$$
\left\{\begin{array}{l}
\frac{\partial u}{\partial t}=\frac{\partial v}{\partial y} \\
\frac{\partial v}{\partial t}=\frac{\partial u}{\partial y}
\end{array}\right.
$$

Here $G(u, v)=(v, u), D G(u, v)=\left[\begin{array}{ll}0 & 1 \\ 1 & 0\end{array}\right], \lambda_{1}=1, \lambda_{2}=-1$, and we choose 
$V_{1}=\left[\begin{array}{l}1 \\ 1\end{array}\right], \quad V_{1}=\left[\begin{array}{c}1 \\ -1\end{array}\right], \quad H(t, y, u, v)=\chi_{1}(t, y, u, v) V_{1}+\chi_{2}(t, y, u, v) V_{2}$ Conditions $\left(1.5 .2^{\prime \prime}\right)$, which are equivalent to (1.4.2), in our case have the form

$$
\left\{\begin{array}{l}
-\frac{\partial \chi_{1}}{\partial u}+\frac{\partial \chi_{1}}{\partial v}=0 \\
\frac{\partial \chi_{2}}{\partial u}+\frac{\partial \chi_{2}}{\partial v}=0
\end{array}\right.
$$

By virtue of $\left(1.5 .1^{\prime}\right) \chi_{1}(t, y, u, v)=h_{1}(t+y, u, v)$ and $\chi_{2}(t, y, u, v)=$ $h_{2}(y-t, u, v)$, where $h_{1}, h_{2} \in C^{1}\left(R^{2}, R\right)$. Substituting these functions into the above system, we obtain

$$
\left\{\begin{array}{l}
-\frac{\partial h_{1}}{\partial u}(t+y, u, v)+\frac{\partial h_{1}}{\partial v}(t+y, u, v)=0 \\
\frac{\partial h_{2}}{\partial u}(y-t, u, v)+\frac{\partial h_{2}}{\partial v}(y-t, u, v)=0
\end{array}\right.
$$

that is, $h_{1}(z, u, v)=\bar{h}_{1}(z, u+v)$ and $h_{2}(z, u, v)=\bar{h}_{2}(z, u-v)$, where functions $\bar{h}_{1}, \bar{h}_{2} \in C^{1}\left(R^{2}, R\right)$. Finally,

$$
H(t, y, u, v)=\left[\begin{array}{l}
\bar{h}_{1}(t+y, u+v)+\bar{h}_{2}(y-t, u-v) \\
\bar{h}_{1}(t+y, u+v)-\bar{h}_{2}(y-t, u-v)
\end{array}\right] .
$$

We thus obtained all solutions of (1.4.1) and (1.4.2). We have to choose such functions $H$ which generate implicitly a $C^{1}$-function, in particular, we have to assume that $\operatorname{det} D_{(u, v)} H \neq 0$. We have $\left(\operatorname{det} D_{(u, v)} I\right)(t, y, u, v)=$ $4 \frac{\partial \bar{h}_{1}}{\partial s}(t+y, u+v) \cdot \frac{\partial \bar{h}_{2}}{\partial s}(y-t, u-v)$, where $\frac{\partial}{\partial s}$ denotes the partial derivative with respect to the second variable $\left(\bar{h}_{i}=\bar{h}_{i}(z, s)\right)$. Now, $I I(t, y, u, v)=\left[\begin{array}{l}c_{1} \\ c_{1}\end{array}\right]$ if and only if $\bar{h}_{1}(t+y, u+v)=\frac{1}{2}\left(c_{1}+c_{2}\right)$ and $\bar{h}_{2}(y-t, u-v)=\frac{1}{2}\left(c_{1}-c_{2}\right)$. We see, that all functions $H$ found above generate implicitly such functions $U(t, y)=\left[\begin{array}{l}u(t, y) \\ v(t, y)\end{array}\right]$ that $u+v=\varphi(t+y)$ and $u-v=\psi(y-t)$, where $\varphi, \psi \in C^{1}(R)$. The functions:

$$
\begin{aligned}
& u(t, y)=\frac{1}{2}[\varphi(t+y)+\psi(y-t)] \\
& v(t, y)=\frac{1}{2}[\varphi(t+y)-\psi(y-t)],
\end{aligned}
$$

where $\varphi, \psi \in C^{1}(R)$, form the set of all $C^{1}$ solutions of our system.

\subsection{A nonlinear system}

Let us consider now the following system for positive functions $u, v$

$$
\left\{\begin{array}{l}
\frac{\partial u}{\partial t}=\frac{\partial}{\partial y}\left(v^{3}\right) \\
\frac{\partial v}{\partial t}=\frac{\partial}{\partial y}\left(u^{3}\right)
\end{array}\right.
$$


Here we have

$$
\begin{gathered}
G(u, v)=\left[\begin{array}{l}
v^{3} \\
u^{3}
\end{array}\right], \quad D G(u, v)=\left[\begin{array}{cc}
0, & 3 v^{2} \\
3 u^{2}, & 0
\end{array}\right], \\
\lambda_{1}(u, v)=3 u v, \quad \lambda_{2}(u, v)=-3 u v \\
V_{1}(u, v)=\left[\begin{array}{l}
v \\
u
\end{array}\right] \quad \text { and } \quad V_{2}(u, v)=\left[\begin{array}{c}
-v \\
u
\end{array}\right], \\
D V_{1}=\left[\begin{array}{ll}
0 & 1 \\
1 & 0
\end{array}\right] \quad \text { and } \quad D V_{2}=\left[\begin{array}{cc}
0,-1 \\
1 & 0
\end{array}\right] .
\end{gathered}
$$

Now the decomposition (1.5) yields

$$
H(t, y, u, v)=\chi_{1}(t, y, u, v)\left[\begin{array}{l}
v \\
u
\end{array}\right]+\chi_{2}(t, y, u, v)\left[\begin{array}{c}
-v \\
u
\end{array}\right]
$$

Equations (1.5.2") in this case have the form

$$
\left\{\begin{aligned}
2 u v\left(-v \frac{\partial \chi_{1}}{\partial u}+u \frac{\partial \chi_{1}}{\partial v}\right) & =-\left(u^{2}-v^{2}\right) \chi_{1}+\left(u^{2}+v^{2}\right) \chi_{2} \\
2 u v\left(v \frac{\partial \chi_{2}}{\partial u}+u \frac{\partial \chi_{2}}{\partial v}\right) & =-\left(v^{2}-u^{2}\right) \chi_{1}-\left(u^{2}+v^{2}\right) \chi_{2}
\end{aligned}\right.
$$

From (1.5.1) it follows that

$$
\begin{aligned}
& \chi_{1}(t, y, u, v)=h_{1}(3 u v t+y, u, v), \\
& \chi_{2}(t, y, u, v)=h_{2}(y-3 u v t, u, v),
\end{aligned}
$$

where $h_{1}, h_{2} \in C^{1}\left(R^{3}\right)$ are arbitrary. If we substitute the functions (2.2.3) into equations (2.2.2), we obtain the following conditions:

$$
\begin{aligned}
& (z-w)\left(u^{2}-v^{2}\right) \frac{\partial h_{1}}{\partial z}(z, u, v)-2 u v\left(v \frac{\partial h_{1}}{\partial u}(z, u, v)-\right. \\
& \left.-u \frac{\partial h_{1}}{\partial v}(z, u, v)\right)+\left(u^{2}-v^{2}\right) h_{1}(z, u, v)=\left(u^{2}+v^{2}\right) h_{2}(w, u, v) \\
& (w-z)\left(u^{2}+v^{2}\right) \frac{\partial h_{2}}{\partial w}(w, u, v)+2 u v\left(v \frac{\partial h_{2}}{\partial u}(w, u, v)+\right. \\
& \left.\quad+u \frac{\partial h_{2}}{\partial v}(w, u, v)\right)+\left(u^{2}+v^{2}\right) h_{2}(w, u, v)=\left(u^{2}-v^{2}\right) h_{1}(z, u, v) .
\end{aligned}
$$

We obtain these conditions by laborious differentiation and then we substitute $z=3 u v t+y$ and $w=y-3 u v t$. Since $z, w, u, v$ may vary independently, we can separate variables. Fixing $u, v, z$ in equation (2.2.4) and varying $w$, we deduce that $h_{1}, h_{2}$ satisfy (2.2.4) if and only if there exist functions 
$P_{2}, Q_{2} \in C^{2}\left(R^{2}, R\right)$ such that the following relations hold:

(i) $h_{2}(w, u, v)=w P_{2}(u, v)+Q_{2}(u, v)$,

(ii) $\frac{\partial h_{1}}{\partial z}(z, u, v)=-\frac{u^{2}+v^{2}}{u^{2}-v^{2}} P_{2}(u, v)$,

(iii) $z\left(u^{2}-v^{2}\right) \frac{\partial h_{1}}{\partial z}-2 u v\left(v \frac{\partial h_{1}}{\partial u}-u \frac{\partial h_{1}}{\partial v}\right)+\left(u^{2}-v^{2}\right) h_{1}=$ $\left(u^{2}+v^{2}\right) Q_{2}(u, v)$

Fixing $u, v, w$ in the condition (2.2.5) and varying $z$, one can find out that $h_{1}, h_{2}$ satisfy condition (2.2.5) if and only if there exist functions $P_{1}, Q_{1} \in$ $C^{1}\left(R^{2}, R\right)$ such that

$$
\begin{aligned}
& \text { (i) } h_{1}(z, u, v)=z P_{1}(u, v)+Q_{1}(u, v), \\
& \text { (ii) } \frac{\partial h_{2}}{\partial w}(w, u, v)=-\frac{u^{2}-v^{2}}{u^{2}+v^{2}} P_{1}(u, v), \\
& \text { (iii) } w\left(u^{2}+v^{2}\right) \frac{\partial h_{2}}{\partial w}+2 u v\left(v \frac{\partial h_{2}}{\partial u}+u \frac{\partial h_{2}}{\partial v}\right)+\left(u^{2}+v^{2}\right) h_{2}= \\
& =\left(u^{2}-v^{2}\right) Q_{1} .
\end{aligned}
$$

Combining conditions (2.2.6) and (2.2.7), we obtain the following system of equations for $P_{1}, Q_{1}, P_{2}$ and $Q_{2}$ :

$$
\begin{aligned}
& \text { (i) } P_{1}(u, v)=-\frac{u^{2}+v^{2}}{u^{2}-v^{2}} P_{2}(u, v) \\
& \text { (ii) }-u v\left(v \frac{\partial P_{1}}{\partial u}-u \frac{\partial P_{1}}{\partial v}\right)+\left(u^{2}-v^{2}\right) P_{1}=0 \\
& \text { (iii) } u v\left(v \frac{\partial P_{2}}{\partial u}+u \frac{\partial P_{2}}{\partial v}\right)+\left(u^{2}+v^{2}\right) P_{2}=0 \\
& \text { (iv) }-2 u v\left(v \frac{\partial Q_{1}}{\partial u}-u \frac{\partial Q_{1}}{\partial v}\right)+\left(u^{2}-v^{2}\right) Q_{1}=\left(u^{2}+v^{2}\right) Q_{2} \\
& \text { (v) } 2 u v\left(v \frac{\partial Q_{2}}{\partial u}+u \frac{\partial Q_{2}}{\partial v}\right)+\left(u^{2}-v^{2}\right) Q_{2}=\left(u^{2}-v^{2}\right) Q_{1}
\end{aligned}
$$

Substituting the function $P_{1}$ from (2.2.8)(i) into the equation (2.2.8)(ii) and then multiplying the result by $-\left(u^{2}-v^{2}\right)\left(u^{2}+v^{2}\right)^{-1}$, we obtain that the resulting equation together with equation (2.2.8)(iii) form a system equivalent to the following

$$
\begin{aligned}
& \text { (i) } v \frac{\partial P_{2}}{\partial v}+u \frac{\partial P_{2}}{\partial u}=0 \\
& \text { (ii) } v \frac{\partial P_{2}}{\partial v}+\frac{u^{2}+v^{2}}{u^{2}-v^{2}} P_{2}=0
\end{aligned}
$$


From 2.2.9(i) it follows that $P_{2}(u, v)=p_{2}\left(\frac{v}{u}\right)$, where $p_{2}$ is a function of class $C^{1}$ of one variable. A function $P_{2}$ satisfies both equations of the system (2.2.9) if, in addition,

$$
p_{2}^{\prime}(x)=-\frac{1+x^{2}}{x\left(1-x^{2}\right)} p_{2}(x)
$$

We integrate this ordinary differential equation and finally we find the entire space of solutions of system 2.2.8(i), (ii) and (iii)

$$
P_{1}(u, v)=-A \frac{u^{2}+v^{2}}{u v}, \quad P_{2}(u, v)=A \frac{u^{2}-v^{2}}{u v}, \quad A \in R .
$$

Let us turn our attention to the system 2.2.8 (iv and v) with unknown functions $Q_{1}$ and $Q_{2}$. This system may be simplified if we introduce new unknown functions $q_{1}(u, v)=\sqrt{u v} Q_{1}(u, v)$ and $q_{2}(u, v)=\sqrt{u v} Q_{2}(u, v)$. Then $Q_{1}, Q_{2}$ satisfy equations $2.2 .8(\mathrm{iv}, \mathrm{v})$ if and only if the pair $q_{1}, q_{2}$ is a solution of the system below

$$
\begin{aligned}
-v \frac{\partial q_{1}}{\partial u}+u \frac{\partial q_{1}}{\partial v} & =\frac{u^{2}+v^{2}}{2 u v} q_{2}, \\
v \frac{\partial q_{2}}{\partial u}+u \frac{\partial q_{2}}{\partial v} & =\frac{u^{2}-v^{2}}{2 u v} q_{1} .
\end{aligned}
$$

Changing variables by means of the diffeomorphism $\varphi: R_{+}^{2} \ni(u, v) \mapsto\left(u^{2}+v^{2}, u^{2}-v^{2}\right)=(r, s) \in\left\{(r, s) \in R^{2}:\left\{(r, s) \in R^{2}: r>|s|\right\}\right.$, i.e., introducing new unknown functions $\widetilde{q}_{1}=q_{1} \circ \varphi^{-1}, \widetilde{q}_{2}=q_{2} \circ \varphi^{-1}$, we see that $\left(q_{1}, q_{2}\right)$ is a solution of $(2.2 .11)$ if and only if $\widetilde{q}_{1}(r, s), \widetilde{q}_{2}(r, s)$ satisfy the following equations:

$$
\begin{aligned}
& \text { (i) } \frac{\partial \widetilde{q_{1}}}{\partial s}=\frac{-r}{2\left(r^{2}-s^{2}\right)} \widetilde{q}_{2}, \\
& \text { (ii) } \frac{\partial \widetilde{q}_{2}}{\partial r}=\frac{s}{2\left(r^{2}-s^{2}\right)} \widetilde{q}_{1} \text {. }
\end{aligned}
$$

To solve the above system, it suffices to find solutions of a single second order hyperbolic equation

$$
\frac{\partial}{\partial r}\left(\frac{r^{2}-s^{2}}{r} \frac{\partial \widetilde{q}_{1}}{\partial s}\right)+\frac{s}{4\left(r^{2}-s^{2}\right)} \widetilde{q}_{1}=0
$$

with one unknown function $\widetilde{q}_{1}$ and then $\widetilde{q}_{2}$ is uniquely determined by $\frac{\partial \tilde{q}_{1}}{\partial s}$. Here $r, s$ are canonical variables of the above hyperbolic equation.

Summarizing (2.2.3), (2.2.6(i)), (2.2.7(i)), (2.2.10-12), we calculated all solutions of the system (1.4.1-2) for the considered equations $(2.2 .1)$ in the 
following form

$$
\begin{array}{r}
H(t, y, u, v)=a\left[\begin{array}{l}
3 t v^{3}+y u \\
3 t u^{3}+y v
\end{array}\right]+\frac{1}{\sqrt{u v}} \tilde{q}_{1}\left(u^{2}+v^{2}, u^{2}-v^{2}\right)\left[\begin{array}{l}
u \\
v
\end{array}\right] \\
-\frac{8(u v)^{3 / 2}}{u^{2}+v^{2}} \cdot \frac{\partial \widetilde{q}_{1}}{\partial s}\left(u^{2}+v^{2}, u^{2}-v^{2}\right)\left[\begin{array}{c}
-v \\
u
\end{array}\right],
\end{array}
$$

where $\widetilde{q}_{1}$ is a solution of equation (2.2.13) and $a=-2 A$. Then 2 -dimensional surfaces $\left\{(t, y, u, v) \in R^{2} \times R_{+}^{2} \mid H(t, y, u, v)=C\right\}, C \in R^{2}$, are integral surfaces of system (2.2.1) and their parts, which are graphs of functions $(t, y) \mapsto(u(t, y), v(t, y))$, define classical solutions of (2.2.1).

\subsection{Some remarks on possible generalizations}

The methods described in Section 1 may also be adapted to more general quasilinear system, namely,

$$
\frac{\partial U}{\partial t}=M(t, y, U(t, y)) \frac{\partial U}{\partial y}+F(t, y, U(t, y)),
$$

where $M$ is a matrix-valued function of $m+2$ variables $t, y, u_{1}, \ldots, u_{m}$ and $F$ has values in $R^{m}$. We then obtain instead of $(1.4 .1-2)$ the following system of linear equations

$$
\begin{gathered}
\frac{\partial H}{\partial t}=M \frac{\partial H}{\partial y}-D_{u} H \cdot F, \\
{\left[M, D_{u} H\right]=0 .}
\end{gathered}
$$

If we assume that there exist sufficiently smooth real valued functions $\lambda_{j}(t, y, u), j=1, \ldots, m$ and $R^{m}$-valued functions $V_{j}(t, y, u), j=1, \ldots, m$, such that $M V_{j}=\lambda_{j} V_{j}$ and, moreover, that eigenvectors $\left\{V_{1}(t, y, u), \ldots\right.$, $\left.\ldots, V_{m}(t, y, u)\right\}$ form a basis of $R^{m}$ for any $(t, y, u)$, then $H$ uniquely defines functions $\chi_{1}, \ldots, \chi_{m}$ of variables $(t, y, u)$ such that

$$
H=\sum_{j=1}^{m} \chi_{j} V_{j}
$$

Reasoning as previously, we obtain the following system instead of (1.5.1)

$$
\frac{\partial \chi_{j}}{\partial t}-\lambda_{j} \frac{\partial \chi_{j}}{\partial y}+D_{u} \chi_{j} \cdot F=\sum_{k=1}^{m} \alpha_{k j} \chi_{k}, \quad j=1, \ldots, m .
$$

The coefficients $\alpha_{k j}(t, y, u)$ are compositions of polynomials with $D V_{1}, \ldots$, $\ldots, D V_{m}, F, M$ and depend also on $V_{1}, \ldots, V_{m}$. If $F=0, \frac{\partial V_{j}}{\partial t}=0, \frac{\partial V_{j}}{\partial y}=0$ for all $j$ 's then all coefficients $\alpha_{k j}$ vanish and system (2.3.5) simplifies, but it may be different than (1.5.1), since eigenvalues $\lambda_{j}$ may still depend on $t, y$. 
Let us point out, that for any classical solution $U$ of (1.1) (or 2.3.1) there exists a function $H$ generating $U$ (by the equation $H=0$ ) and satisfying (1.4.2) (or (2.3.3), respectively), but the equation 1.4.1 (respectively, (2.3.2)) may, perhaps, be satisfied only in points $(t, y, u) \in R^{2} \times R^{m}$ such that $H(t, y, u)=0$. On the other hand, taking $m$-parameter families of solutions in the form $H(t, y, u)=C$, where $C$ takes values in some open subset of $R^{m}$ and $\left[M, D_{u} H\right]=0$, we see that $H$ satisfies (1.4.1) (or (2.3.2), respectively) on some open subset in $R^{2} \times R^{m}$.

Can we actually organize all classical solutions of systems (2.3.1) in such families $H$ that $M$ and $D_{u} H$ commute? It seems doubtful for me. Systems (2.3.2-3) and also (1.4.1-2), if considered as systems of partial differential equations for $H$ defined on an open subset in $R^{2} \times R^{m}$, are very restrictive in general. There is also a possible way of generalization of the method. We can require that $\left[D_{u} H, M\right]=M_{1} D_{u} H$ instead of (2.3.3), where $M_{1}$ is an arbitrary matrix-valued function. Then we also obtain equation (2.3.2) by substituting $M+M_{1}$ in place of $M$ in it.

\section{Barotropic fluid}

We shall consider motion of a inviscid barotropic fluid in a ball $K=$ $\left\{x \in R^{n} \mid\|x\|<r_{0}\right\}, r_{0}>0$. Let

$$
v:[0, T] \times \bar{K} \ni(t, x) \mapsto v(t, x) \in R^{n}
$$

be the velocity vector field of a fluid and let

$$
\varrho:[0, T] \times \bar{K} \ni(t, x) \mapsto \varrho(t, x) \in R_{+}
$$

be the density of mass. The pressure of the barotropic fluid is described by the function $p=f \circ \varrho$, where $f: R_{+} \rightarrow R$ is a given $C^{1}$-function with positive derivative, i.e. $f^{\prime}(y)>0$ for $y>0$.

The equations of fluid motion can be written as follows.

$$
\begin{aligned}
& \frac{\partial v}{\partial t}+D_{x} v \cdot v=-\frac{1}{\varrho}\left(f^{\prime} \circ \varrho\right) \nabla \varrho, \\
& \frac{\partial \varrho}{\partial t}+\operatorname{div}(\varrho v)=0,
\end{aligned}
$$

where $\left(D_{x} v\right)(t, \cdot)=D(v(t, \cdot))$ is the derivative of $v(t, \cdot): K \rightarrow R^{n},(\nabla \varrho)(t, \cdot)$ $=[D(\varrho(t, \cdot))]^{T}$ and "div" means the divergence operator for vector fields on $R^{n}$, that is, the divergence with respect to variables $\left(x_{1}, \ldots, x_{n}\right)$. The pair $(\varrho, v)$ is a classical solution of the equation of motion if $\varrho$ and $v$ are continuous on $[0, T] \times \bar{K}$ and of class $C^{1}$ on $(0, T) \times K$, they satisfy (3.1.1-2) in every point $(t, x) \in(0, T) \times K$ and, moreover, $v$ is tangent to the boundary $\partial K$, i.e., $v$ satisfies the boundary condition 


$$
\begin{array}{r}
\langle v(t, x), x\rangle=0 \text { for every }(t, x) \in[0, T] \times\left\{x \in R^{n} \mid\|x\|=r_{0}\right\}, \\
\text { where }\langle., .\rangle \text { is the inner product of } R^{n} .
\end{array}
$$

The initial data consist of a function $\varrho_{0}: \bar{K} \rightarrow R$ and a vectorfield $v_{0}: \bar{K} \rightarrow R^{n}$, which is tangent to the boundary $\partial K$. It is known (see Serrin [4]) that for sufficiently smooth $\varrho_{0}, v_{0}$ there exist at most one solution of (3.1.1-3) satisfying the initial conditions

$$
\varrho(0, x)=\varrho_{0}(x), \quad v(0, x)=v_{0}(x), \quad x \in \bar{K} .
$$

Let us consider two actions $\Phi, \Phi_{0}$ of the orthogonal group $O(n)$ on the spaces of continuous maps $C\left([0, T] \times \bar{K}, R \times R^{n}\right)$ and $C\left(\bar{K}, R \times R^{n}\right)$, respectively, defined as follows

$$
\Phi_{0}\left(A,\left(\varrho_{0}, v_{0}\right)\right)=\left(\varrho_{0}^{A}, v_{0}^{A}\right), \quad \Phi(A,(\varrho, v))=\left(\varrho^{A}, v^{A}\right), \quad A \in O(n),
$$

where

$$
\begin{gathered}
\varrho_{0}^{A}(x)=\varrho_{0}(A x), \quad v_{0}^{A}(x)=A^{-1} v_{0}(A x), \quad x \in \bar{K} \\
\left(\varrho^{A}(t, x), v^{A}(t, x)\right)=\left(\varrho(t, A x), A^{-1} v(t, A x)\right), \quad(t, x) \in[0, T] \times \bar{K} .
\end{gathered}
$$

3.2.1. Proposition. The space of classical solutions of (3.1.1-3) is $O(n)$ invariant with respect to the (right) action $\Phi$.

P r o of. We have the following derivatives of $\varrho^{A}$ and $v^{A}$

$$
\begin{gathered}
\left(\nabla \varrho^{A}\right)(t, x)=A^{T}(\nabla \varrho)(t, A x)=A^{-1}(\nabla \varrho)(t, A x) \\
\frac{\partial \varrho^{A}}{\partial t}(t, x)=\frac{\partial \varrho}{\partial t}(t, A x), \quad\left[\left(D_{x} v^{A}\right) \cdot v^{A}\right](t, x)=A^{-1}\left(\left(D_{x} v\right) v\right)(t, A x) \\
\frac{\partial v^{A}}{\partial t}(t, x)=A^{-1} \frac{\partial v}{\partial t}(t, A x) \quad \text { and } \quad \operatorname{div}\left(\varrho^{A} v^{A}\right)(t, x)=(\operatorname{div}(\varrho v)(t, A x)
\end{gathered}
$$

for $\varrho, v$ of class $C^{1}$ on $(0, T) \times K$. Substituting these derivatives into (3.1.1-2) it is easy to see that $\left(\varrho^{A}, v^{A}\right)$ satisfies the equations of motion if and only if $(\varrho, v)$ does. The boundary condition (3.1.3) is also satisfied

$$
\left\langle x, v^{A}(t, x)\right\rangle=\left\langle x, A^{-1} v(t, A x)\right\rangle=\left\langle x, A^{T} v(t, A x)\right\rangle=\langle A x, v(t, A x)\rangle=0
$$

for $x \in \partial K$.

$\mathrm{Re} \mathrm{m}$ a r k. If we replace $K$ by $R^{n}$, we can extend the action on $G L(n, R)$ (by means of the same formula (3.1.5)). In this case the space of solutions of the continuity equation (3.1.2) is $G L(n, R)$-invariant, but the space of solutions of (3.1.1-2) is not $G L(n, R)$-invariant, it is still $O(n)$-invariant.

A solution of (3.1.1-3) is said to be symmetric with respect to $G \subset O(n)$, where $G$ is a subgroup, if $G$ is the isotropy group of $\Phi$ for this solution, i.e., 
$\left(\varrho^{A}, v^{A}\right)=(\varrho, v)$ if and only if $A \in G$. Similarly, we say that initial data $\left(\varrho_{0}, v_{0}\right)$ are $G$-symmetric if $G$ is the isotropy group of $\Phi_{0}$ at $\left(\varrho_{0}, v_{0}\right)$.

3.2.2. Proposition. If the initial data $\left(\varrho_{0}, v_{0}\right)$ are $G$-symmetric and there exists a solution $(\varrho, v)$ of $(3.1 .1-4)$, then $(\varrho, v)$ is symmetric with respect to $G$. And conversely: any $G$-symmetric solution of initial problem (3.1.1-4) is originated from the $G$-symmetric initial data.

Proof. This fact follows easily from Proposition 3.2.1 and the uniqueness theorem for solutions to the initial problem.

$\mathrm{R}$ e $\mathrm{m}$ ark. The results formulated in Propositions 3.2.1-2 are surely well known, but we find it easier to prove them than to find them in a text-book.

All $O(n)$-symmetric initial data have the form:

$$
\begin{cases}\varrho_{0}(x)=\gamma_{0}(|x|), & \gamma_{0}:\left[0, r_{0}\right] \ni y \rightarrow \gamma_{0}(y) \in R_{+} \\ v_{0}(x)=\mu_{0}(|x|) x, & \mu_{0}:\left[0, r_{0}\right] \ni y \rightarrow \mu_{0}(y) \in R\end{cases}
$$

and, similarly, for any solution of (3.1.1-2) symmetric with respect to $O(n)$ there exist continuous functions $\gamma, \mu:[0, T] \times\left[0, r_{0}\right] \rightarrow R$ such that

(i) $\varrho(t, x)=\gamma(t,|x|), v(t, x)=\mu(t,|x|) x$,

(ii) $\gamma$ is of class $C^{1}$ on $(0, T) \times\left[0, r_{0}\right)$

$$
\text { and } \frac{\partial \gamma}{\partial y}(t, 0)=0 \text { for any } t \in(0, T)
$$

(iii) $\mu$ is of class $C^{1}$ on $(0, T) \times\left[0, r_{0}\right)$

$$
\text { and } \frac{\partial \mu}{\partial y}(t, 0)=0 \text { for any } t \in(0, T) .
$$

The functions $\gamma(t, y)>0$ and $\mu(t, y)$ defined by an $O(n)$-symmetric solution of the initial problem (3.1.1-3.1.4) are solutions of the following initial problem

$$
\begin{array}{ll}
\text { (3.4.1) } & \frac{\partial \mu}{\partial t}+\mu^{2}+y \mu \frac{\partial \mu}{\partial y}=\frac{-1}{y \gamma}\left(f^{\prime} \circ \gamma\right) \frac{\partial \gamma}{\partial y}, \\
\text { (3.4.2) } & \frac{\partial \gamma}{\partial t}+n \mu \gamma+y \frac{\partial(\gamma \mu)}{\partial y}=0 \\
\text { (3.4.3) } & \mu\left(t, r_{0}\right)=0 \quad \text { for every } t \in[0, T], \\
\text { (3.4.4) } & \gamma(0, y)=\gamma_{0}(y), \quad \mu(0, y)=\mu_{0}(y) \quad \text { for every } y \in\left[0, r_{0}\right]
\end{array}
$$

Each equation (3.4.k) is derived from (3.1.k), $k=1, \ldots, 4$. 


\section{Flows symmetric with respect to $O(n)$}

We first show how the system (3.4.1-2) may be simplified.

Let $f_{0}(y)=\int_{1}^{y} \frac{f^{\prime}(s)}{s} d s$. Multiplying equation (3.4.1) by $y$ and multiplying equation (3.4.2) by $y^{n-1}$ and then introducing new unknown functions $w(t, y)=y \mu(t, y), z(t, y)=y^{n-1} \gamma(t, y)$, we obtain

$$
\begin{aligned}
& \frac{\partial w}{\partial t}=\frac{\partial}{\partial y}\left(-\frac{1}{2} w^{2}-f_{0}\left(y^{1-n} z\right)\right) \\
& \frac{\partial z}{\partial t}=\frac{\partial}{\partial y}(-w z)
\end{aligned}
$$

that is, the system has the form $\frac{\partial}{\partial t} U=\frac{\partial}{\partial y} G(y, U)$, where $U(t, y)=\left[\begin{array}{l}w(t, y) \\ z(t, y)\end{array}\right]$.

4.2. Proposition. For every local solution $(w(t, y), z(t, y))$ of system (4.1.1-2) with positive $z$, defined on a simply-connected domain, there exists $a C^{2}$ function $u(t, y)$ on the same domain satisfying the following equation

$$
\begin{aligned}
u_{y}^{2} u_{t t}-2 u_{t} u_{y} u_{t y}+\left[u_{t}^{2}-u_{y}^{2} f^{\prime}\left(y^{1-n} u_{y}\right)\right] u_{y y}+ & \\
& +\frac{n-1}{y} u_{y}^{3} f^{\prime}\left(y^{1-n} u_{y}\right)=0,
\end{aligned}
$$

where $u_{t}, u_{y}$ denote first order partial derivatives of $u$ and $u_{t t}, u_{t y}, u_{y y}$ are second order partial derivatives of $u$ with respect to $t, y$, respectively, and $u$ defines $w, z$ as follows

$$
z=u_{y}, \quad w=-\frac{u_{t}}{u_{y}} .
$$

P r o of. If $(z, w)$ is a $C^{1}$ solution of 4.1 .2 on a simply-connected domain, then, by virtue of Poincaré Lemma, there exists a $C^{2}$ function $u$ such that $z=u_{y}$ and $-w z=u_{t}$. Since $z$ is assumed to be positive, we see that (4.2.2) holds. Substituting this function into (4.1.1) and multiplying the result by $-u_{y}^{3}$, we obtain (4.2.1).

Thus, equation (4.2.1), which is a quasilinear second order hyperbolic equation with a single unknown real function, generates (by formula 4.2.2) all symmetric flows

$$
\varrho(t, x)=|x|^{1-n} u_{y}(t,|x|), \quad v(t, x)=\frac{-u_{t}(t,|x|)}{|x| u_{y}(t,|x|)} x,
$$

We have also the converse statement.

4.3. Proposition. If $u$ is a $C^{2}$ solution of equation (4.2.1) on $(0, T) \times$ $\left(0, r_{0}\right)$ such that $u_{y}$ is positive and $\varrho, v$ defined by (4.2.3) are of class $C^{1}$ on $(0, T) \times K$, then these $\varrho$ and $v$ satisfy the equations of motion (3.1.1-2). 
Proof. If $u_{y}>0$ and $u$ is a solution of (4.2.1), then

$$
u_{y}^{3} \frac{\partial}{\partial t}\left(\frac{u_{t}}{u_{y}}\right)-\frac{\partial}{\partial y}\left(\frac{u_{t}^{2}}{2 u_{y}^{2}}+f_{0}\left(y^{1-n} u_{y}\right)\right)=0
$$

since the left hand side of the above is just equal to the left hand side of (4.2.1). Dividing by $u_{y}^{3}$ and denoting $u_{y}=z,-u_{t} / u_{y}=w$, we see that $w, z$ satisfy (4.1.1-2). Hence $\mu=\frac{1}{y} w$ and $\gamma=y^{1-n} z$ form a pair of functions fulfilling equations (3.4.1-2). This implies in turn that $(\varrho, v)$ defined by $(4.2 .3)$ is an $O(n)$-symmetric solution of (3.1.1-2).

Let us also point out that two solutions of (4.2.1) define the same symmetric flow if and only if they differ by a constant.

\section{Symmetric flows defined implicitly}

We assume now that the pressure is proportional to the density of mass. This means that $f(y)=c^{2} y$, where $c>0$ is a constant, which has a physical interpretation, namely, $c$ is the sound speed in fluid. In this section we apply the method described in Sections 1 and 2 to the system (4.1.1-2). We rewrite this system in the form (2.3.1)

$$
\frac{\partial}{\partial t}\left[\begin{array}{l}
z \\
w
\end{array}\right]=-\left[\begin{array}{cc}
w, & z \\
c^{2} / z, & w
\end{array}\right] \frac{\partial}{\partial y}\left[\begin{array}{l}
z \\
w
\end{array}\right]+\left[\begin{array}{c}
0 \\
(n-1) c^{2} / y
\end{array}\right] .
$$

We are seeking $R^{2}$-valued functions $H(t, y, z, w)$ of four variables defining implicitly solutions of $(5.1)$ by equality $H(t, y, z, w)=\left[\begin{array}{l}c_{1} \\ c_{2}\end{array}\right]$. The eigenvalues $\lambda_{1}(t, y, w, z)$ and $\lambda_{2}(t, y, z, w)$ of the matrix $M(t, y, z, w)$ are: $\lambda_{1}=-w-c$ and $\lambda_{2}=-w+c$ and the corresponding smooth eigenvector fields are

$$
V_{1}=\left[\begin{array}{l}
z \\
c
\end{array}\right], \quad V_{2}=\left[\begin{array}{c}
z \\
-c
\end{array}\right]
$$

Thus any $H$ can be written as follows

$$
H(t, y, z, w)=\chi(t, y, z, w)\left[\begin{array}{l}
z \\
c
\end{array}\right]+\theta(t, y, z, w)\left[\begin{array}{c}
z \\
-c
\end{array}\right]
$$

Since

$$
D_{(z, w)} H \cdot F=\left[\begin{array}{cc}
z \chi_{z}+z \theta_{z}+\chi+\theta, & z \chi_{w}+z \theta_{w} \\
c \chi_{z}-c \theta_{z}, & c \chi_{w}-c \theta_{w}
\end{array}\right] \cdot\left[\begin{array}{c}
0 \\
\frac{(n-1) c^{2}}{y}
\end{array}\right],
$$

where subscripts stand for first order partial derivatives with respect to corresponding variables, we obtain

$$
D_{(z, w)} H \cdot F=\frac{(n-1) c^{2}}{y} \chi_{w}\left[\begin{array}{l}
z \\
c
\end{array}\right]+\frac{(n-1) c^{2}}{y} \theta_{w}\left[\begin{array}{c}
z \\
-c
\end{array}\right] .
$$


Therefore, the equations corresponding to (2.3.2) or, equivalently, to (2.3.5) may be written
a) $\chi_{t}+(w+c) \chi_{y}+\frac{(n-1) c^{2}}{y} \chi_{w}=0$
b) $\theta_{t}+(w-c) \theta_{y}+\frac{(n-1) c^{2}}{y} \theta_{w}=0$.

Condition (2.3.3) leads to the system
a) $z \chi_{z}-c \chi_{w}+\frac{1}{2} \chi+\frac{1}{2} \theta=0$,
b) $z \theta_{z}+c \theta_{w}+\frac{1}{2} \chi+\frac{1}{2} \theta=0$,

which is equivalent to the condition $\left[D_{(z, w)} H, M\right]=0$.

Let us point out here that if $H$ satisfies both systems (5.4.1-2) and $\operatorname{det} D_{(z, w)} H \neq 0$, then $H$ generates families of solutions of system (4.1.1-2) with $f_{0}(y)=c^{2} \ln y\left(\right.$ for $\left.f^{\prime}(y)=c^{2}\right)$.

Conversely, for any solution of (4.1.1-2) there exist functions $\chi, \theta$ such that $\chi(t, y, z, w)=0, \theta(t, y, z, w)=0$ defines this solution, $(\chi, \theta)$ satisfies (5.4.2) on $(0, T) \times\left(0, r_{0}\right) \times R^{2}$ and equations (5.4.1) are satisfied in points $(t, y, z, w)$ on this integral surface.

We shall study the solutions of system (5.4.1-2).

5.5. THEOREM. All solutions of system (5.4.1-2) which are defined on $(0, T) \times\left(0, r_{0}\right) \times R_{+} \times R$ have the form

$$
\begin{aligned}
& \chi(t, y, z, w)=A\left[\ln \left(y^{1-n} z\right)+\frac{1}{2}\left(\frac{w}{c}+1\right)^{2}\right]+\frac{D}{z}-C \\
& \theta(t, y, z, w)=-A\left[\ln \left(y^{1-n} z\right)+\frac{1}{2}\left(\frac{w}{c}-1\right)^{2}\right]+\frac{D}{z}+C,
\end{aligned}
$$

where $A, D, C \in R$.

P r o of. We first integrate (5.4.1b) and we get

$$
\begin{aligned}
& \theta(t, y, z, w)= \\
& \tilde{h}\left(z, y\left(g^{\prime}\left(\frac{2}{c} w\right)\right)^{-1},-2(n-1) c t+y g\left(\frac{2}{c} w\right)\left(g^{\prime}\left(\frac{2}{c} w\right)\right)^{-1}\right),
\end{aligned}
$$

where

$$
g(x)=\frac{1}{2(n-1) c} \int_{0}^{x} \exp \frac{(s / 2-1)^{2}}{2(n-1)} d s,
$$

and $\widetilde{h}(\alpha, \beta, \zeta)$ is a $C^{1}$-function on a suitable open subset of $R^{3}$. Let $\hat{\chi}=\sqrt{z} \chi$ and $\hat{\theta}=\sqrt{z} \theta$. Then $(\chi, \theta)$ satisfies (5.4.2) if and only if $(\hat{\chi}, \hat{\theta})$ satisfies the 
following system

$$
\begin{aligned}
& z \widehat{\chi}_{z}-c \hat{\chi}_{w}+\frac{1}{2} \hat{\theta}=0, \\
& z \hat{\theta}_{z}+c \widehat{\theta}_{w}+\frac{1}{2} \hat{\chi}=0 .
\end{aligned}
$$

This system can be further simplified if we introduce new variables $r=z \cdot \exp (w / c)$ and $s=z \cdot \exp (-w / c)$. Then $z=\sqrt{r s}$ and $w=\frac{c}{2} \ln \frac{r}{s}$ and for

$$
\begin{aligned}
& \tilde{\chi}(t, y, r, s):=\hat{\chi}\left(t, y, \sqrt{r s}, \frac{c}{2} \ln \frac{r}{s}\right) \\
& \tilde{\theta}(t, y, r, s):=\hat{\theta}\left(t, y, \sqrt{r s}, \frac{c}{2} \ln \frac{r}{s}\right),
\end{aligned}
$$

we obtain equations:
(a) $\tilde{\chi}_{s}=\frac{-1}{4 s} \tilde{\theta}$
(b) $\tilde{\theta}_{r}=\frac{-1}{4 r} \tilde{\chi}$

or, equivalently,
(a) $\tilde{\boldsymbol{\theta}}_{r s}-\frac{1}{16 r s} \tilde{\boldsymbol{\theta}}=0$
(b) $\tilde{\chi}=-4 r \tilde{\theta}_{r}$.

Now, by virtue of equality $(5.5 .1)$, the function $\tilde{\theta}$ can be written

$$
\tilde{\theta}(t, y, r, s)=h\left(r s, \frac{y}{g^{\prime}(\ln (r / s))},-2(n-1) c t+\frac{y g(\ln (r / s))}{g^{\prime}(\ln (r / s))}\right),
$$

where $h(\alpha, \beta, \zeta)=\widetilde{h}(\sqrt{\alpha}, \beta, \zeta) \alpha^{1 / 4}$.

Substituting $\tilde{\theta}$ expressed by (5.5.3) into (5.5.2'(a)) and then multiplying by $r s$, we obtain

$$
\left(\alpha^{2} h_{\alpha \alpha}+\alpha h_{\alpha}-\frac{1}{16} h+\frac{\beta}{4(n-1)} h_{\beta}\right)+\frac{\left(\frac{q}{2}-1\right)^{2}}{4(n-1)^{2}}\left(-\beta^{2} h_{\beta \beta}-\beta h_{\beta}\right)+
$$

$$
\begin{aligned}
& \left.+g^{\prime \prime}(q)\left(2 \beta^{2} h_{\beta \zeta}+\beta h_{\zeta}\right)\right)+\frac{\left(\frac{q}{2}-1\right)^{2}}{4(n-1)^{2}} g(q)\left(-2 \beta^{2} h_{\beta \zeta}-\beta h_{\zeta}\right)+ \\
& +\frac{1}{4(n-1)} g(q) \beta h_{\zeta}-\beta^{2}\left(g^{\prime}(q)-g^{\prime \prime}(q) \frac{g(q)}{g^{\prime}(q)}\right)^{2} h_{\zeta \zeta}=0
\end{aligned}
$$

where all derivatives of $h$ are taken in a point $(\alpha, \beta, \zeta)$, which can be expressed by $t, y, r, s$ as in (5.5.3) and $q=\ln (r / s)$. Let us notice, that we can fix $(\alpha, \beta, \zeta)$ at will and, independently, we can vary the $q$. Since the functions of $q$ (written in the above equality as coefficients) are independent, we 
conclude that equation (5.5.2') implies the following system of equations for $h$ appearing in (5.5.3):

$$
\begin{aligned}
& \text { a) } \alpha^{2} h_{\alpha \alpha}+\alpha h_{\alpha}-\frac{1}{16} h+\frac{\beta}{4(n-1)} h_{\beta}=0, \\
& \text { b) } \beta^{2} h_{\beta \beta}+\beta h_{\beta}=0 \\
& \text { c) } h_{\zeta}=0 .
\end{aligned}
$$

From (5.5.5 b,c) we obtain

$$
h(\alpha, \beta, \zeta)=f_{1}(\alpha)+f_{2}(\alpha) \ln \beta,
$$

where $f_{1}, f_{2}$ are functions of a single variable. Equation (5.5.5a) gives us, in turn, a system of 2 ordinary differential equations for $f_{1}, f_{2}$ of Euler type

$$
\left\{\begin{array}{l}
\alpha^{2} f_{1}^{\prime \prime}(\alpha)+\alpha f_{1}^{\prime}(\alpha)-\frac{1}{16} f_{1}(\alpha)=\frac{-1}{4(n-1)} f_{2}(\alpha) \\
\alpha^{2} f_{2}^{\prime \prime}(\alpha)+\alpha f_{2}^{\prime}(\alpha)-\frac{1}{16} f_{2}(\alpha)=0 .
\end{array}\right.
$$

which has solutions

$$
\left\{\begin{array}{l}
f_{1}(\alpha)=\frac{1}{2}\left[-A \alpha^{1 / 4} \ln \alpha+B \alpha^{-1 / 4} \ln \alpha\right]+C_{1} \alpha^{1 / 4}+D_{1} \alpha^{-1 / 4} \\
f_{2}(\alpha)=(n-1)\left[A \alpha^{1 / 4}+B \alpha^{-1 / 4}\right]
\end{array}\right.
$$

Thus all solutions of $\left(5.5 .2^{\prime} \mathrm{a}\right)$ satisfying (5.5.3) are

$$
\begin{aligned}
\widetilde{\theta}(t, y, r, s)= & f_{1}(r s)-f_{2}(r s) \cdot \ln \left(\frac{1}{y}\left(g^{\prime}(\ln r / s)\right)\right)= \\
= & \frac{1}{2}\left[B(r s)^{-1 / 4}-A(r s)^{1 / 4}\right] \ln (r s)+C(r s)^{1 / 4}+D(r s)^{-1 / 4}+ \\
& +\left[A(r s)^{1 / 4}+B(r s)^{-1 / 4}\right]\left[\ln y^{n-1}-\frac{1}{2}\left(\frac{1}{2} \ln \frac{r}{s}-1\right)^{2}\right]
\end{aligned}
$$

Substituting the above $\tilde{\theta}$ into $\left(5.5 .2^{\prime} \mathrm{b}\right)$, we calculate all $\tilde{\chi}$ corresponding to $\tilde{\theta}$ in system (5.5.2). Then we come back to the previous functions and variables

$$
\begin{aligned}
& \chi(t, y, z, w)=\frac{1}{\sqrt{z}} \tilde{\chi}\left(t, y, z e^{w / c}, z e^{-w / c}\right), \\
& \theta(t, y, z, w)=\frac{1}{\sqrt{z}} \tilde{\theta}\left(t, y, z e^{w / c}, z e^{-w / c}\right) .
\end{aligned}
$$

When we substitute $\chi$ (calculated from $\tilde{\theta}$ ) into (5.4.1a), we see that this equation is satisfied if and only if the coefficient $B$ vanishes. Then we obtain the final result. 
By virtue of Theorem 5.5 and formula (5.2), we obtain all functions $I I$ on $(0, T) \times\left(0, r_{0}\right) \times R_{+} \times R$ satisfying equations $(2.3 .2-3)$ for system (5.1):

$$
H(t, y, z, w)=\left[\begin{array}{l}
K_{1}+K_{3} z w \\
K_{2}+K_{3}\left(\frac{1}{2} w^{2}+c^{2} \ln \left(y^{1-n} z\right)\right)
\end{array}\right],
$$

where $K_{1}=2 D, K_{2}=-2 c C+A c$ and $K_{3}=2 A / c$ are real constants. This set of solutions is very small. We can identify functions $H$ and $\varphi \circ H$, where $\varphi$ is any diffeomorphism of $R^{2}$, because $H$ and $\varphi \circ H$ define the same family of 2-dimensional integral surfaces in $R^{4}$. In fact, all functions in (5.6) belong to one class of equivalent maps, which may be represented by a single map $H_{0}$ :

$$
H_{0}(t, y, z, w)=\left[\begin{array}{l}
z w \\
\frac{1}{2} w^{2}+c^{2} \ln \left(y^{1-n} z\right)
\end{array}\right],
$$

and Jacobian det $D_{(z, w)} H_{0}=0$ if and only if $w^{2}=c^{2}$.

The equality $H_{0}(t, y, z, w)=\left[\begin{array}{l}c_{1} \\ c_{2}\end{array}\right]$ generates a graph of a function

$$
(0, T) \times\left(0, r_{0}\right) \ni(t, y) \rightarrow(z(t, y), w(t, y)) \in R_{+} \times R
$$

if and only if $c_{1}=0$. We then obtain $w(t, y)=0$ and $z(t, y)=K y^{n-1}$, where $K$ is a positive constant, i.e., $H_{0}$ generates the 1-parameter family of trival $O(n)$-symmetric solutions: $\varrho(t, x)=K, v(t, x)=0$. This shows that the nonlinear nature of barotropic flows is very deep (see [3]).

\section{Acknowledgement}

I am very grateful to Professor Erhard Meister for the helpful hints and several improvements introduced into the manuscript of this paper.

\section{References}

[1] D. G. Ebin, The initial boundary value problem for sub-sonic fluid motion, Comm. Pure Appl. Math. 32 (1979), 1-19.

[2] T. Kato, The Cauchy problem for quasi-linear symmetric hyperbolic systems, Arch. Rational Mech. Anal. 58 (1975), 181-205.

[3] C. Marchioro, M. Pulvirenti, Mathematical theory of incompressible viscous fluids, Springer-Verlag, 1994.

[4] J. Serrin, On the uniqueness of compressible fluid motions, Arch. Rational Mech. Anal. 3 (1959), 271-288.

INSTITUTE OF MATHEMATICS

WARSAW UNIVERSITY OF TECHNOLOGY

P1. Politechniki 1

00-661 WARSZAWA, POLAND

Received May 18, 1994. 

